\title{
Quantificação das Placas Coronarianas Calcificadas pela Tomografia Computadorizada do Tórax: Correlação com a Técnica do Escore de Cálcio
}

\author{
Quantification of Calcified Coronary Plaques by Chest Computed Tomography: Correlation with the Calcium \\ Score Technique
}

Vitor Frauches Souza, ${ }^{1,2}$ Alair Augusto Sarmet M. D. dos Santos, ${ }^{1,2}$ Claudio Tinoco Mesquita, ${ }^{2}$ Wolney de Andrade Martins, ${ }^{2 \bullet}$ Gustavo Lemos Pelandre, ${ }^{3 \oplus}$ Edson Marchiori, ${ }^{3}$ Marcelo Souto Nacif ${ }^{4,5}$

Complexo Hospitalar de Niterói - Radiologia, ${ }^{1}$ Niterói, RJ - Brasil

Hospital Universitário Antônio Pedro - Pós-Graduação em Ciências Cardiovasculares, ${ }^{2}$ Niterói, RJ - Brasil

Universidade Federal do Rio de Janeiro - Pós-Graduação em Radiologia, ${ }^{3}$ Rio de Janeiro, RJ - Brasil

Universidade Federal Fluminense - Pós-graduação em Ciências Cardiovasculares, ${ }^{4}$ Niterói, RJ - Brasil

Complexo Hospitalar de Niterói, ${ }^{5}$ Niterói, $R J$ - Brasil

\section{Resumo}

Fundamento: A doença cardiovascular representa a principal causa de mortalidade no mundo. Calcificações parietais nas artérias podem ser visualizadas e quantificadas por tomografia computadorizada (TC) em estágios iniciais e subclínicos, sendo expressa em escore de cálcio (EC). Com esse número, é possível estimar o prognóstico de eventos cardiovasculares futuros.

Objetivos: Correlacionar a detecção e quantificação do EC pela TC do tórax utilizando como padrão-ouro a TC cardíaca sincronizada ao eletrocardiograma.

Métodos: Estudo transversal e descritivo que selecionou pacientes $(n=73)$ consecutivos para investigação de doença arterial coronariana estável e que realizaram TC cardíaca no período de junho de 2013 a outubro de 2014 . Realizado protocolo com TC do tórax e EC, em aparelho de 64 canais. Os valores de $p<0,05$ foram considerados estatisticamente significativos.

Resultados: Na avaliação por paciente, após a transformação logarítmica a média do EC sincronizado foi de 8,7 e na TC de tórax foi de 9,4. Prevalência de doença de 49,3\% $(n=36)$. A sensibilidade foi de $97,2 \%$ e a especificidade de $100,0 \%$. Observou-se excelente correlação entre os métodos $(r=0,993 \mathrm{com} p<0,001)$. Na avaliação por segmento, a média do EC sincronizado foi de 3,0. Já a média do EC na TC de tórax foi de 3,2. Prevalência de doença de 29,5\% ( $\mathrm{n}=86$ ), com sensibilidade de $95,3 \%$ e especificidade de $97,5 \%$. Observou-se também excelente correlação entre os métodos $(r=$ $0,985$ com $\mathrm{p}<0,001)$.

Conclusão: O EC sincronizado e não sincronizado têm boa correlação entre si e não mostram resultados estatisticamente diferentes. (Arq Bras Cardiol. 2020; 115(3):493-500)

Palavras-chave: Doença da Artéria Coronariana; Placa Aterosclerótica; Calcificação Vascular; Rigidez Vascular; Pontuação de Propensão; Tomografia Computadorizada por Raios-X /métodos.

\footnotetext{
Abstract

Background: Cardiovascular disease is the leading cause of mortality in the world. Parietal calcifications of the arteries may be visualized and quantified at initial and subclinical states by computed tomography (CT), and expressed as calcium score (CS). It is possible to estimate the prognosis of future cardiovascular events using this score.
}

Objectives: To correlate the detection and quantification of the CS obtained by chest CT with that obtained by electrocardiography (ECG)synchronized cardiac computed tomography (the gold-standard).

Method: Cross-sectional, descriptive study of 73 consecutive patients in investigation for coronary artery disease who underwent cardiac CT between June 2013 and October 2014. Chest computed tomography and CS protocols were performed in a 64-channel TC scanner. P-values $<0.05$ were considered statistically significant.

Correspondência: Vitor Frauches Souza •

Complexo Hospitalar de Niterói - Radiologia - Rua La Salle 12. CEP 24020-096, Centro, Niterói, RJ - Brasil

E-mail: drvitorfrauches@gmail.com

Artigo recebido em 06/08/2019, revisado em 27/07/2019, aceito em 18/08/2019

DOI: https://doi.org/10.36660/abc.20190235 
Results: In the per-patient analysis, after logarithmic transformation, mean CS was 8.7 and 9.4 by the ECG-synchronized method and chest CT, respectively. The prevalence of disease was $49.3 \%(n=36)$, with a sensitivity of $97.2 \%$ and specificity of $100.0 \%$. There was an excellent correlation between the methods ( $r=0.993, p<0.001)$. In the per-segment analysis, after logarithmic transformation, mean CS was 3.0 and 3.2 by the ECG-synchronized method and chest CT, respectively. The prevalence of disease was $29.5 \%(n=86)$, with a sensitivity of $95.3 \%$ and specificity of $97.5 \%$. There was an excellent correlation between the methods $(r=0.985, p<0.001)$.

Conclusion: ECG-synchronized CT is well correlated with the non-ECG-synchronized CT for CS determination, without statistical difference between the methods. (Arq Bras Cardiol. 2020; 115(3):493-500)

Keywords: Coronary Artery Disease; Plaque,Atherosclerotic; Vascular Calcification; Vascular Stiffness; Tomography, X-Ray/ methods

Full texts in English - http://www.arquivosonline.com.br

\section{Introdução}

A doença cardiovascular é a principal causa de mortalidade no mundo. Segundo a Organização Mundial da Saúde, apenas no ano de 2011, ocorreram 17 milhões de mortes decorrentes de doenças cardiovasculares, das quais 7 milhões secundárias à doença aterosclerótica coronariana (DAC) e 6,2 milhões causadas pelas doenças vasculares cerebrais. ${ }^{1}$ Estima-se que o número de mortes por doença cardiovascular chegará a 23,3 milhões em 2030, mantendo-se entre as principais causas de morte. ${ }^{2}$

O gasto anual com o tratamento da DAC é alto e envolve métodos de imagem invasivos e não-invasivos, novos medicamentos, tratamentos endovasculares e mesmo cirúrgicos, o que tem sobrecarregado cada vez mais os apertados orçamentos para a saúde. A descoberta de métodos com melhor custo-eficácia para o diagnóstico de DAC tem atraído investimentos e ajudado no rápido avanço tecnológico nesta área, contribuindo de forma exponencial para a eficácia dos tratamentos e do manejo clínico desta doença. Neste contexto, o escore de cálcio (EC) tem papel cada vez mais importante no diagnóstico da DAC., ${ }^{3,4}$ O EC já foi demonstrado como marcador independente de risco para eventos cardiovasculares e mortalidade cardíaca. Além disso também provém informações adicionais de prognóstico para outros marcadores de risco cardiovascular. ${ }^{5}$

Muitos pacientes realizam tomografia computadorizada (TC) do tórax para avaliação de diversas síndromes clínicas, como dispneia e dor torácica, assim como de possível pneumonia, massa mediastinal ou pulmonar, trauma, entre outras. Esses pacientes poderiam se beneficiariam de uma estratificação de risco para doença cardiovascular, possibilitando a ampliação de intervenções de prevenção primária e secundária. Um dado relevante é que somente nos EUA, o EC poderia potencialmente ser relatado em aproximadamente 7,1 milhões de exames de TC sem contraste realizados anualmente. ${ }^{6}$

Este estudo objetiva correlacionar a detecção e quantificação do EC pela TC do tórax não sincronizada ao ECG utilizando como padrão-ouro a TC cardíaca sincronizada ao ECG.

\section{Pacientes e métodos}

Foi realizado um estudo transversal e descritivo, que selecionou pacientes em investigação de DAC e que realizaram TC cardíaca no período de junho de 2013 a outubro de 2014, de modo consecutivo. O tamanho da amostra foi por conveniência e teve relação com o número de exames realizados na unidade hospitalar no período estipulado para o trabalho. A indicação dos exames ficou a critério do médico assistente, não sendo objetivo do estudo a análise das indicações.

Os pacientes elegíveis foram submetidos a um protocolo específico para a realização da TC de tórax simples, não sincronizada, e avaliação do EC por TC cardíaca acoplada ao ECG em sessão única, sem alteração de posicionamento, com as menores doses de radiação possíveis, moduladas pelo próprio aparelho. Foram utilizados cortes de 1,0 mm de espessura na TC do tórax e para o EC cortes axiais sequenciais, sem espaçamento e com $3 \mathrm{~mm}$ de espessura cobrindo toda a extensão do coração, sendo este o método padrão da prática clínica.

A voltagem do tubo em todos os exames foi de $120 \mathrm{Kv}$ e sua corrente variável de acordo com a modulação do aparelho, ficando entre 150 e $400 \mathrm{~mA}$, seguindo os protocolos recomendados pelo fabricante e utilizados na instituição. Ambos os exames foram realizados no mesmo tomógrafo de 64 canais Sensation 64 (Siemens, Hanover, Alemanha). Todos os pacientes com frequência cardíaca acima de 70 batimentos por minuto receberam terapia betabloqueadora intravenosa com $5 \mathrm{mg}$ a 50mg de tartarato de metoprolol antes da aquisição das imagens.

\section{Cálculo do EC}

A análise do cálcio coronariano foi feita por meio do EC de Agatston (aquisição sincronizada e não sincronizada ao ECG), utilizando o software Ca Scoring (Siemens, Hanover, Alemanha), pela análise quantitativa semiautomática. Foram considerados focos de calcificação áreas com atenuação igual ou superior a 130 unidades Hounsfield e área igual ou superior a 3 pixel e pelo menos $1 \mathrm{~mm}^{2}$, e marcadas com sinal colorido.

A análise das artérias coronárias foi dividida em quatro ramos principais: tronco da artéria coronária esquerda (TCE), artéria descendente anterior (ADA), artéria circunflexa (ACX) e artéria coronária direita (ACD), e assim foi atribuído o escore, que é resultado do produto entre a densidade e sua área de calcificação. O EC total é resultado da soma dos escores individuais de cada região. Para cada ramo arterial foi quantificado o número de placas e o escore de Agatston.

\section{Análise estatística}

Todas as variáveis contínuas foram expressas como média \pm desvio padrão e as categóricas como número e percentual. Foram considerados os seguintes intervalos de valores de 
calcificação: zero (ausência de calcificação); maior do que zero e menor do que 100 (calcificação leve); maior do que 100 e menor do que 400 (calcificação moderada); maior do que 400 (calcificação grave).

Foi utilizado o teste t de Student para dados pareados para determinar se os resultados obtidos pelo cálculo EC eram significativamente diferentes dos obtidos pela TC do tórax na avaliação global (por paciente). Além disso, foram realizadas comparações por segmentos de cada território coronariano divididos em: TCE, ADA, ACx e ACD. As análises do EC foram tratadas com log $(E C+1)$ para corrigir os desvios desta amostra quando necessário.

Realizou-se a análise do coeficiente de classificação de Spearman (rho) para detectar o grau de correlação entre o EC gerado pela técnica sincronizada e pela TC do tórax quanto os seguintes estratos: 0 (ausência de calcificação); 0 - 100 (calcificação leve); 100 - 400 (calcificação moderada); maior que 400 (calcificação grave).

Recorreu-se à regressão linear e ao coeficiente de correlação de Pearson ( $r$ ) para avaliar a correlação entre o EC pela técnica sincronizada e não sincronizada (TC de tórax) considerando: correlação ausente se $r=$ ZERO; fraca se $r=0,01-0,20$; baixa se $r=0,21-0,40$; moderada se $r=0,41-0,60$; boa se $r=0,61-0,80$; e excelente se $r=0,81-1,00$. Também, realizamos Bland-Altman para demonstrar a variabilidade (viés) e os limites de concordância (intervalo de confiança de 95\%) entre as técnicas.

Analisou-se o TCE, a ADA, a ACx e a ACD de cada paciente, totalizando 288 vasos pelo EC somado com a TC do tórax pelo observador 1. Aproximadamente 50\% dos exames, ou seja, 36 pacientes e 144 segmentos, foram reavaliados pelo mesmo observador, dando maior força para os resultados encontrados. Também foram realizadas análises por um segundo observador independente, de maneira totalmente cega ao primeiro observador. Além disso, o observador 2 repetiu esta análise após o período de 3 meses de forma totalmente cega à primeira análise. A concordância intraobservador e interobservador foi obtida usando-se a análise da confiabilidade das médias do coeficiente de correlação intraclasse $(\mathrm{CCl}<0,40$ fraca concordância; $\mathrm{CCl}$ $=0,40-0,59$ moderada concordância; $\mathrm{CCl}=0,60-0,74$ boa concordância; $\mathrm{CCl}=0,75-1,00$ excelente concordância). Reiteramos que as análises foram totalmente cegas e baseadas na experiência de ambos os observadores. Vale frisar que o observador 1 possui 2 anos de experiência e o observador 2 acima de 12 anos.

Análise de curva característica de operação do receptor (receiver operating characteristic, ROC) foi utilizada para identificar o desempenho diagnóstico da TC de tórax (não sincronizada) para predizer o EC (sincronizado) por paciente e por segmento. Isto foi realizado com uso dos grupos 0 - 100 (calcificação leve); 101 - 400 (calcificação moderada); maior que 400 (calcificação grave) como marcadores substitutos de "verdadeiro positivo" nesta população, em comparação com o grupo com 0 (ausência de calcificação) como o "verdadeiro negativo" ( $A \cup C \geq 0,5$ e $<0,7=$ ajuste pobre, $A \cup C \geq 0,7$ e $<0,9=$ bom ajuste e AUC $\geq 0,9$ e 1,0= excelente ajuste).

Para a análise estatística foi utilizado o MedCalc ${ }^{\circledR}$, versão 17.8 (MedCalc Software bvba, Ostend, Bélgica). Os valores de $p<0,05$, bicaudais, foram considerados estatisticamente significativos.
O estudo foi aprovado pela comissão de ética da instituição sede do estudo, sob o número 771.854. O estudo seguiu os preceitos éticos determinados pela Declaração de Helsinque. Todos os pacientes participantes assinaram o termo de consentimento livre e esclarecido, tendo sido garantidas a retirada do consentimento e a exclusão do estudo a qualquer momento, a pedido do indivíduo ou de seu responsável, sem prejuízo à assistência, confidencialidade, sigilo ou privacidade.

\section{Resultados}

De um total de 82 pacientes, foram incluídos no estudo 73 pacientes, 37 (51,4\%) do sexo masculino, com a média de idade de 58,9 $\pm 13,1$ anos, entre junho de 2013 e outubro de 2014. Durante a amostragem foram excluídos do estudo nove pacientes, sendo quatro pela presença de stents coronarianos, um por enxerto de mamária, e outros quatro por dificuldades técnicas tais como artefatos de movimento que impediram a análise das imagens e problemas de envio das sequências adquiridas para o servidor de imagens.

\section{Análise por paciente}

Após a transformação logarítmica, observou-se que o método não sincronizado (TC de tórax) superestima discretamente os valores obtidos pelo sincronizado, com médias de 9,4 e 8,7, respectivamente.

Foi averiguada excelente correlação entre os métodos. Achados estes que podem ser observados na Figura 1.

Dos 73 pacientes estudados, 37 apresentaram EC (sincronizado) igual a zero; 18 com EC entre 0 - 100; sete pacientes com EC entre 100 - 400; e 11 pacientes com EC $>$ 400. Pela TC de tórax (não sincronizada) o EC igual a zero (ausência de calcificação) foi encontrado em 35 indivíduos; 19 apresentaram EC entre 0 - 100 (calcificação leve); 6 tiveram o EC entre 100 - 400 (calcificação moderada); e 13 com EC $>400$ (calcificação grave). A correlação do coeficiente de classificação de Spearman (rho) para classificação dos grupos acima foi de 0,96. A Tabela 1 sintetiza os achados da análise por paciente.

\section{Análise segmentar}

A média do EC sincronizado (método padrão) na análise segmentar foi de 50,1土179,7 Agatston e sua média após transformação logarítmica foi de 3,0 Agatston com intervalo de confiança de $95 \%$ de 2,4 a 3,8. Já a média do EC na

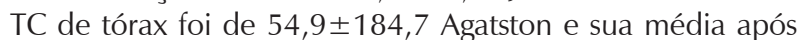
transformação logarítmica foi de 3,2 Agatston com intervalo de confiança de $95 \%$ de 2,5 a 4,1.

Na Figura 2, pode-se observar que foi encontrada excelente correlação entre os métodos.

Na análise segmentar, 292 segmentos foram incluídos, sendo que 206 apresentaram EC igual a zero; 56 com EC entre 0 - 100; 21 segmentos com EC entre 100 - 400; nove segmentos com EC > 400. Pela TC de tórax não sincronizada, o EC igual a zero foi encontrado em 197; em 59 segmentos foi encontrado EC entre 0 - 100; EC entre 100 - 400 em 25 segmentos; e 11 com EC > 400. A correlação do coeficiente 


\section{Artigo Original}


Figura 1 - Análise por paciente. (A) Regressão linear demonstrando a correlação entre o método de tomografia computadorizada de tórax não sincronizada com ecocardiografia (ECG) e o método de tomografia computadorizada cardíaca sincronizada com ECG. (B) Análise de Bland-Altman com os dados obtidos por ambos os métodos. A diferença média é representada pela linha azul e o limite de concordância pelo traçado vermelho; TC: tomografia computadorizada do tórax; EC: escore de cálcio.

Tabela 1 - Distribuição dos pacientes pela classificação de escore de cálcio pelas técnicas de tomografia computadorizada sincronizada e não sincronizada com ecocardiografia, na análise por paciente $(n=73)$

\begin{tabular}{lllll}
\hline & $\begin{array}{l}\text { Técnica } \\
\text { sincronizada }\end{array}$ & Escore de Cálcio & $\begin{array}{l}\text { Técnica não } \\
\text { sincronizada }\end{array}$ & Escore de cálcio \\
\hline Grupos & $\mathrm{N}(\%)$ & Média e DP & $\mathrm{N}(\%)$ & Média e DP \\
0 & $37(50,7)$ & 0 & $35(47,9)$ & 0 \\
0 a 100 & $18(24,7)$ & $24,3 \pm 24,7$ & $19(26,0)$ & $26,0 \pm 34,0$ \\
100 a 400 & $7(9,6)$ & $222,4 \pm 99,8$ & $6(8,2)$ & $166,3 \pm 71,5$ \\
$>400$ & $11(15,1)$ & $1150,7 \pm 980,2$ & $13(17,8)$ & $1118,2 \pm 867,7$ \\
\hline
\end{tabular}

DP: desvio padrão.
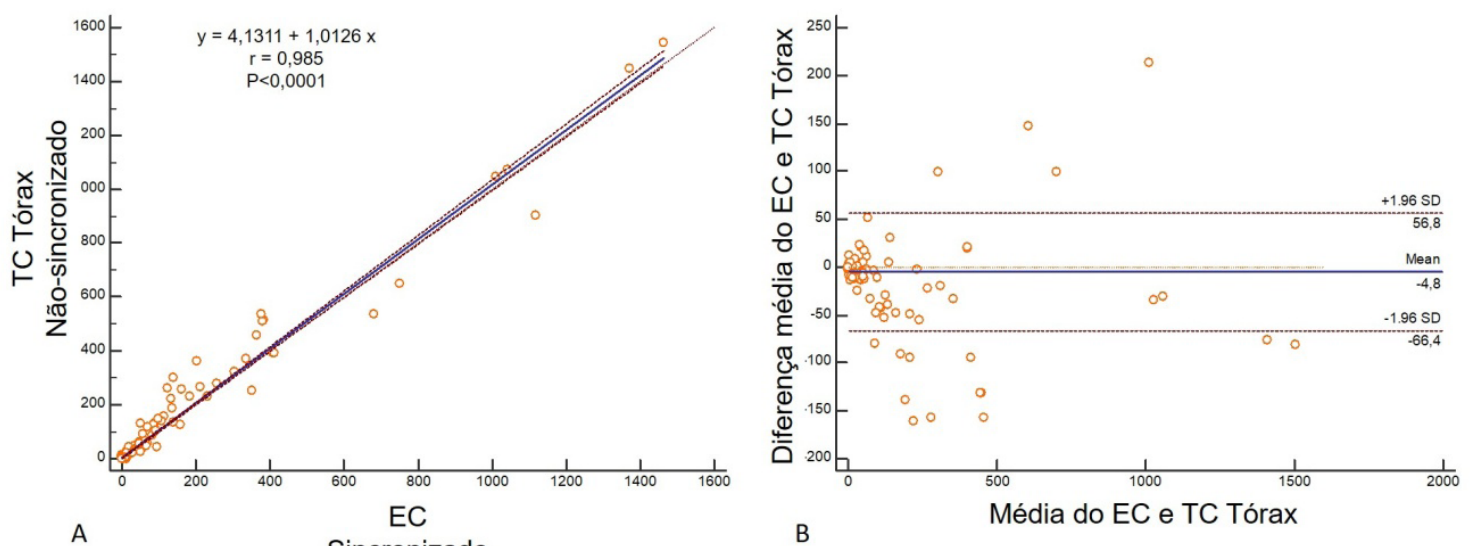

Sincronizado

Figura 2 - Análise por segmento. (A) Regressão linear demonstrando a correlação entre o método de tomografia computadorizada de tórax não sincronizada com ecocardiografia (ECG) e o método de tomografia computadorizada cardíaca sincronizada com ECG. (B) Análise de Bland-Altman com os dados obtidos por ambos os métodos. A diferença média é representada pela linha azul e o limite de concordância pelo traçado vermelho. 
de classificação de Spearman (rho) para classificação segmentar dos grupos anteriormente descritos foi de 0,92. Os achados da análise segmentar estão apresentados na Tabela 2.

\section{Correlação segmentar entre os métodos}

A média do EC pelo método sincronizado com ECG foi 200,7 Agatston, e sua distribuição nas coronárias da seguinte forma: TCE 6,9; ADA 88,7; ACx 26,4 e ACD 88,6. Já a média do EC na TC de tórax foi 219,5 e sua distribuição nas coronárias: TCE 8,4; ADA 85,4; ACx 29,1 e ACD 96,6. Tais dados podem ser mais bem visualizados na Tabela 3 .

\section{Prevalência, especificidade e sensibilidade}

Os dados encontrados de especificidade e sensibilidade tanto para a análise por paciente quanto por segmento estão dispostos na Figura 3.

Na análise por paciente $(n=73)$, com uma prevalência de doença de $49,3 \%(n=36)$, a TC de tórax foi capaz de identificar os pacientes com placas calcificadas com uma área sob a curva (ROC AUC) de 0,99 com intervalo de confiança de $95 \%$ de 0,99 a $1,00(p<0,0001)$. A sensibilidade foi de $97,2 \%$ e a especificidade de $100,0 \%$.

Já na avaliação segmentar $(n=292)$, com uma prevalência de doença de $29,5 \%(n=86)$, a TC de tórax foi capaz de identificar os segmentos com placas calcificadas com uma área sob a curva (ROC_AUC) de 0,98 com intervalo de confiança de $95 \%$ de 0,96 a $1,00(p<0,0001)$. A sensibilidade foi de $95,3 \%$ e a especificidade de $97,5 \%$.
A Figura 4 demonstra três exemplos diferentes de calcificação na artéria descendente anterior, caracterizados pelo EC e pela TC do tórax.

\section{Concordância intraobservador e interobservador}

Excelente concordância intraobservador e interobservador foi demonstrada na quantificação das placas calcificadas pela técnica do EC e pela técnica da TC de tórax, $\mathrm{com} \mathrm{CCl}>$ 0,99 para todos.

\section{Discussão}

Nos últimos anos o rastreio de pacientes assintomáticos para a descoberta de doença cardiovascular em estágios iniciais tem ganhado grande relevância socioepidemiológica e causado controvérsia no âmbito dos estudos científicos. ${ }^{3,7-9}$

Indubitavelmente a grande quantidade de exames de TC de tórax realizadas por outros propósitos pode auxiliar no acompanhamento cardiovascular de pacientes assintomáticos com o fornecimento de informações clínicas relevantes, evitando-se a repetição de exames para esse fim ou até mesmo selecionando os pacientes que merecem o tratamento adequado.

Desde seu advento, a TC demonstrou ser um ótimo método para detecção de câncer de pulmão e de outras doenças pulmonares. Com os últimos avanços, foi possível a realização de exames de coração sincronizados ao ECG, possibilitando a quantificação de cálcio coronariano e, por conseguinte, estratificação do risco cardiovascular. Nesse contexto, a realização de um único exame de TC em que fosse

Tabela 2 - Distribuição dos pacientes pela classificação de escore de cálcio pelas técnicas de tomografia computadorizada sincronizada e não sincronizada com ecocardiografia, na análise por segmento $(n=292)$

\begin{tabular}{|c|c|c|c|c|}
\hline & $\begin{array}{c}\text { Técnica } \\
\text { sincronizada }\end{array}$ & Escore de Cálcio & $\begin{array}{l}\text { Técnica não } \\
\text { sincronizada }\end{array}$ & Escore de cálcio \\
\hline Grupos & $\mathrm{N}(\%)$ & Média e DP & $\mathrm{N}(\%)$ & Média e DP \\
\hline 0 & $206(70,5)$ & 0 & $197(67,5)$ & 0 \\
\hline 0 a 100 & $56(19,2)$ & $28,6 \pm 29,9$ & $59(20,2)$ & $20,1 \pm 22,6$ \\
\hline 100 a 400 & $21(7,2)$ & $228,4 \pm 101,1$ & $25(8,6)$ & $225,5 \pm 93,0$ \\
\hline$>400$ & $9(3,1)$ & $917,5 \pm 381,7$ & $11(3,8)$ & $838,1 \pm 393,5$ \\
\hline
\end{tabular}

DP: desvio padrão.

Tabela 3 - Correlação por segmentos coronarianos entre o escore de cálcio obtido pelas técnicas de tomografia computadorizada (TC) sincronizada (TC cardíaca) e não sincronizada (TC de tórax) com ecocardiografia

\begin{tabular}{|c|c|c|c|c|c|}
\hline & Escore de cálcio & TC de tórax & Pearson & Teste $\mathrm{t}$ & \\
\hline & Média e DP & Média e DP & $r(95 \% \mathrm{IC})$ & Teste $t(p)$ & N \\
\hline TCE & $6,9 \pm 23,4$ & $8,4 \pm 27,3$ & $0,90(0,85$ a 0,93$)$ & 0,25 & 73 \\
\hline ADA & $88,7 \pm 278,5$ & $85,4 \pm 198,5$ & $0,97(0,96$ a 0,95$)$ & 0,85 & 73 \\
\hline $\mathrm{ACx}$ & $26,4 \pm 75,5$ & $29,1 \pm 78,7$ & $0,98(0,96$ a 0,98$)$ & 0,14 & 73 \\
\hline ACD & $88,6 \pm 278,5$ & $96,6 \pm 293,1$ & $0,99(0,98$ a 0,99$)$ & 0,08 & 73 \\
\hline
\end{tabular}

TCE: tronco da coronária esquerda: $A D A$ : artéria descendente anterior; $A C x$ : artéria circunflexa; $A C D$ : artéria coronária direita. 


\section{Artigo Original}

\section{Quantificação do escore de cálcio pela TC Tórax (não-sincronizada)}
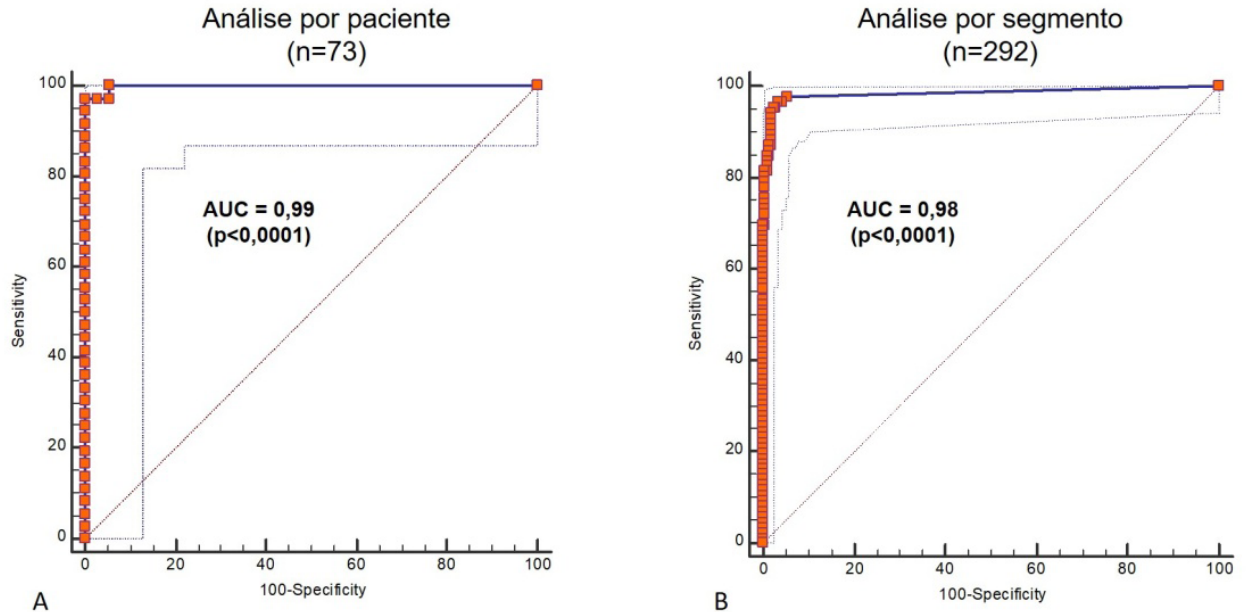

Figura 3 - Análise da curva ROC (receiver operating characteristic) com quantificação da área sob a curva utilizando a tomografia computadorizada do tórax para predizer placa calcificada detectada pelo escore de cálcio; (A) análise por paciente; B) análise por segmento.

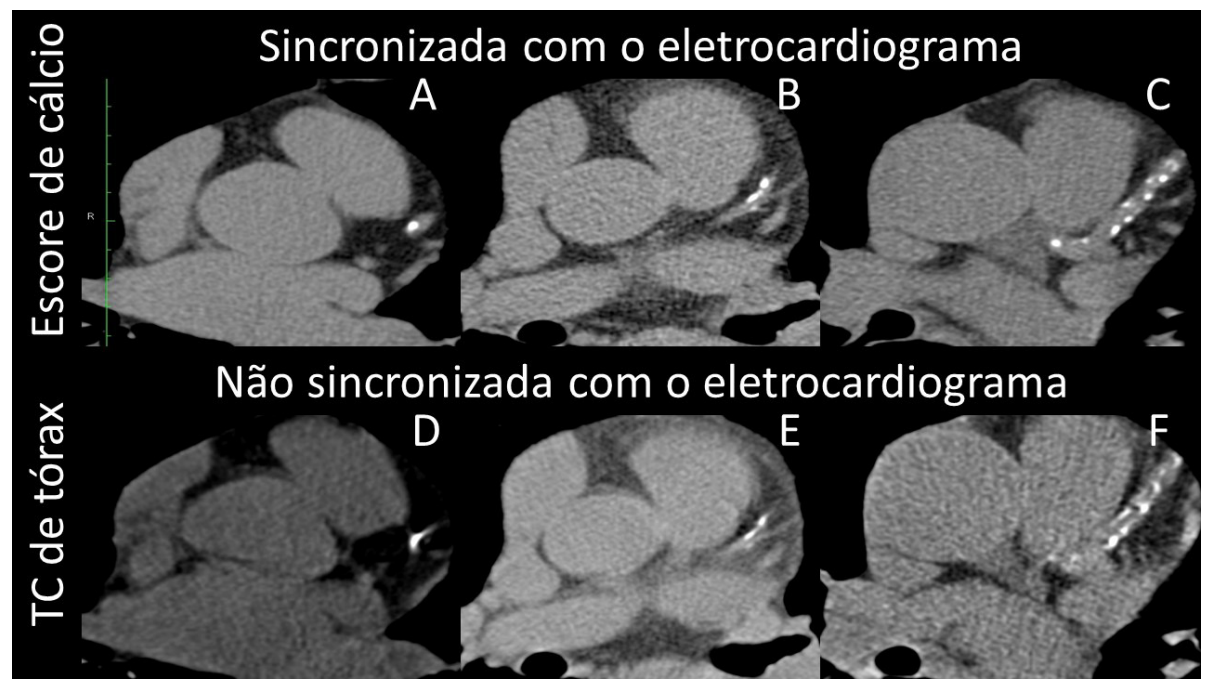

Figura 4 - Exemplos de quantificação pela técnica do escore de cálcio $(A, B$ e $C$ ), sem movimento da coronária. Mesmos pacientes com a quantificação pela tomografia computadorizada de tórax (D, E e F) com um certo grau de movimento da coronária. Observar as placas na descendente anterior: o primeiro caso com pequena calcificação $(A$ e $D)$; um segundo caso com duas calcificações $(B$ e E) e um terceiro caso com múltiplas placas calcificadas ( $C$ e $F$ ).

possível avaliar a presença de tumores pulmonares e o risco cardiovascular teria uma significativa relevância clínica. ${ }^{10-15}$

A TC sincronizada ao ECG ainda é o método referência para identificação e quantificação do cálcio coronariano, com validação estabelecida. No entanto, estudos clássicos com TC do tórax não sincronizada ao ECG também mostraram que a identificação visual (não quantitativa) do cálcio coronariano fornece informações clínicas relevantes. ${ }^{12,15-17}$ Desta forma, é evidente que a avaliação quantitativa do EC na aquisição da TC de tórax pode revelar dados ainda mais importantes e fidedignos.

O grande avanço tecnológico dos tomógrafos nos últimos 20 anos permitiu uma notória melhora na avaliação das calcificações coronarianas nos exames não sincronizados ao ECG, pois os cortes mais finos e a rapidez na aquisição dos dados reduziram significativamente os artefatos produzidos por movimentos cardíacos e por efeito de volume parcial. ${ }^{18}$ 
Nesse espectro, este trabalho objetivou demostrar a relação entre os valores de EC encontrados na tomografia cardíaca sincronizada ao ECG em aparelho de 64 canais de detectores com aqueles vistos em exames do tórax com baixa dose de radiação, realizados no mesmo aparelho, a fim de possibilitar um aumento de informações radiológicas e clínicas nas TCs de tórax realizadas rotineiramente nos hospitais e clínicas.

Foi possível detectar e quantificar o EC pela TC de tórax não sincronizada e, quando se comparou com a técnica padrão-ouro, o EC sincronizado, os resultados foram excelentes. Porém, com a transformação logarítmica dos valores encontrados, observou-se que a TC de tórax superestima discretamente os valores obtidos pelo método de referência $(p=0,0012)$, uma vez que está mais suscetível às alterações causadas por artefatos de movimento respiratório e pelos batimentos cardíacos.

Todas as correlações de Pearson foram superiores a $0,90(p<0,0001)$ na análise por paciente ou segmentar. Há variabilidades entre os métodos, com viés muito pequeno (4,1 Agatston) e achados inferiores a 3,2\% da diferença média entre as técnicas, e esses resultados não são de importância clínica. Esses achados vão ao encontro do estudo de Budoff et al., ${ }^{19}$ demonstrando excelente correlação entre os dois métodos.

A TC de tórax foi capaz de classificar a população conforme as diretrizes atuais, com excelente correlação e sem diferença estatística significativa quando comparada ao método ouro por territórios coronarianos. Os valores encontrados para a correlação de Pearson nesta avaliação variaram entre 0,90 e 0,99 e o p do teste T de student, entre 0,08 e 0,85, conforme os dados disponíveis na Tabela 3.

Em apenas dois casos foram observados falsos positivos, no qual o EC total foi zero na técnica padrão ouro e 0,3 e 0,6 Agatston na TC de tórax. Tal achado pode ter duas explicações plausíveis, a primeira devido à presença de artefatos inerentes ao método e que foram considerados como calcificação pelo software, visto a diminuta carga cálcica nesses dois achados, e a segunda relacionada aos cortes mais finos da TC de tórax $(1 \mathrm{~mm})$ comparados aos do EC (3 mm); desta forma, pequeninas placas antes não identificadas pelo método ouro, poderiam ser identificadas na TC de tórax. Isso abre um questionamento sobre a espessura de corte da técnica padrão do EC ser a mesma desde sua criação por Agatston no início dos anos 90, quando ainda não eram disponíveis aparelhos com tecnologia para cortes mais finos.

Desta forma, incentiva-se o uso, como forma alternativa, da TC de tórax não sincronizada para a detecção do EC. Esse método possui capacidade diagnóstica semelhante ao padrão-ouro, atingindo excelente sensibilidade e especificidade, com área sob a curva superior a 0,98 $(p<0,0001)$, demonstrando assim, sensibilidade de 97,2\% e especificidade de $100,0 \%$ para a análise por paciente e sensibilidade de 95,3\% e especificidade de 97,5\% quanto feito por segmento coronariano. Pelandré et al. ${ }^{20}$ demonstraram a capacidade de utilização do método em um estudo semelhante, com excelente acurácia utilizando software dedicado ou análise visual.
Neste contexto, a sociedade de TC cardiovascular (SCCT - Society for Cardiovascular Computed Tomography) desenvolveu uma diretriz para utilização da TC de tórax na avaliação de placas calcificadas coronarianas, demonstrando a importância da descrição desses achados de forma quantitativa e qualitativa, além da topografia destas placas. ${ }^{21}$

Pelo exposto, demonstra-se o quão importante é para o radiologista a descrição por completo da presença ou não de cálcio nos estudos tomográficos do tórax, e quando possível sua quantificação, visto que o estudo comprovou que a capacidade diagnóstica é excelente mesmo frente a diferença entre os métodos.

\section{Limitação do estudo}

A limitação deste estudo foi em virtude da necessidade de administração de betabloqueador adrenérgico, prática que não é rotineira nas TC de tórax. Acreditamos que pacientes com frequência cardíaca elevada terão maiores artefatos nas imagens não trigadas. Também consideramos de extrema importância a aplicabilidade destes resultados em diferentes centros com diferentes tomógrafos, porém os nossos resultados não devem ser extrapolados sem uma testagem inicial para as outras tecnologias.

\section{Conclusão}

O presente estudo demonstrou excelente correlação entre a quantificação das placas calcificadas pela TC de tórax não sincronizada ao ECG quando comparada ao EC em estudos com a utilização de betabloqueadores.

De posse dos resultados deste estudo, incentivase o uso, como forma alternativa, da TC de tórax não sincronizada para a detecção do EC, a fim de auxiliar no acompanhamento e estratificação do risco de pacientes que muitas vezes não tem acesso a uma tomografia cardíaca.

\section{Contribuição dos Autores}

Concepção e desenho da pesquisa: Souza VF, Nacif MS. Obtenção de dados: Souza VF. Análise e interpretação dos dados: Souza VF. Análise estatística: Souza VF, Nacif MS. Obtenção de financiamento: Souza VF. Redação do manuscrito: Souza VF. Revisão crítica do manuscrito quanto ao conteúdo intelectual importante: Santos AASMD, Mesquita CT, Martins WA, Pelandre GL, Marchiori E, Nacif MS.

\section{Potencial Conflito de Interesse}

Declaro não haver conflito de interesses pertinentes.

\section{Fontes de Financiamento}

O presente estudo foi parcialmente financiado por CNPq.

\section{Vinculação Acadêmica}

Este artigo é parte de dissertação de Mestrado de Vitor Frauches Souza pela Universidade Federal Fluminense. 


\section{Aprovação Ética e Consentimento Informado}

Este estudo foi aprovado pelo Comitê de Ética da Universidade Federal Fluminense sob o número de protocolo

\section{Referências}

1. World Health Organization. (WHO). Causes of death. Global Health Observatory (GHO) data [Internet]. Geneva: WHO; 2016. [citado em 1 Jun 2016]. Disponível em: http://www.who.int/gho/mortality_burden_disease/ causes_death/en

2. Mathers CD, Loncar D. Projections of global mortality and burden of disease from 2002 to 2030. PLoS Med. 2006;3(11):e442.

3. Greenland P, Alpert JS, Beller GA, Benjamin EJ, Budoff MJ, Fayad ZA, et al. $2010 \mathrm{ACCF} / \mathrm{AHA}$ guideline for assessment of cardiovascular risk in asymptomatic adults: a report of the American College of Cardiology Foundation/American Heart Association Task Force on Practice Guidelines. J Am Coll Cardiol. 2010;56(25):e50-103.

4. Taylor AJ, Cerqueira M, Hodgson JM, Mark D, Min J, O'Gara P, et al. ACCF/ SCCT/ACR/AHA/ASE/ASNC/NASCI/SCAI/SCMR 2010 Appropriate Use Criteria for Cardiac Computed Tomography. A Report of the American College of Cardiology Foundation Appropriate Use Criteria Task Force, the Society of Cardiovascular Computed Tomography, the American College of Radiology, the American Heart Association, the American Society of Echocardiography, the American Society of Nuclear Cardiology, the North American Society for Cardiovascular Imaging, the Society for Cardiovascular Angiography and Interventions, and the Society for Cardiovascular Magnetic Resonance. J Cardiovasc Comput Tomogr. 2010;4(6):407.e1-33.

5. Neves PO, Andrade J, Monção H. Coronary artery calcium score: current status. Radiol Bras. 2017;50(3):182-9.

6. Berrington de Gonzalez A, Mahesh M, Kim KP, Bhargavan M, Lewis R, Mettler $\mathrm{F}$, et al. Projected cancer risks from computed tomographic scans performed in the United States in 2007. Arch Intern Med. 2009;169(22):2071-7.

7. Hecht HS. Coronary artery calcium scanning: past, present, and future. JACC Cardiovasc Imag. 2015;8(5):579-96.

8. Agatston AS, Janowitz WR, Hildner FJ, Zusmer NR, Viamonte Jr M, Detrano R. Quantification of coronary artery calcium using ultrafast computed tomography. J Am Coll Cardiol. 1990;15(4):827-32.

9. Bastarrika G, Alonso A, Saiz-Mendiguren R, Arias J, Cosin O. Coronary artery calcium quantification with non-ECG-gated low-dose CT of the chest. Radiologia. 2010;52(1):30-6.

10. Fuster V. Lewis A. Conner Memorial Lecture. Mechanisms leading to myocardial infarction: insights from studies of vascular biology. Circulation. 1994;90(4):2126-46.
771.854/2014. Todos os procedimentos envolvidos neste estudo estão de acordo com a Declaração de Helsinki de 1975, atualizada em 2013. O consentimento informado foi obtido de todos os participantes incluídos no estudo.

11. Badimon JJ, Fuster V, Chesebro JH, Badimon L. Coronary atherosclerosis. a multifactorial disease. Circulation. 1993;87(3 Suppl):II3-16.

12. Kalia NK, Miller LG, Nasir K, Blumenthal RS, Agrawal N, Budoff MJ. Visualizing coronary calcium is associated with improvements in adherence to statin therapy. Atherosclerosis. 2006;185(2):394-9.

13. Jacobs PCA, Isgum I, Gondrie MJA, Mali WPTM, van Ginneken B, Prokop M, et al. Coronary artery calcification scoring in low-dose ungated CT screening for lung cancer: interscan agreement. AJR Am J Roentgenol. 2010;194(5):1244-9.

14. Takx RA, Jong PA, Leiner T, Oudkerk M, Koning HJ, Mol CP, et al. Automated coronary artery calcification scoring in non-gated chest CT: agreement and reliability. PLoS One. 2014;9(3):e91239.

15. Kirsch J, Martinez F, Lopez D, Novaro GM, Asher CR. National trends among radiologists in reporting coronary artery calcium in non-gated chest computed tomography. Int J Cardiovasc Imaging. 2017;33(2):251-7.

16. Shao L, Yan AT, Lebovic G, Wong HH, Kirpalani A, Deva DP. Prognostic value of visually detected coronary artery calcificaton on unenhanced non-gated thoracic computed tomography for prediction of non-fatal myocardial infarction and all-cause mortality. J Cardiovasc Comput Tomogr. 2017;11(3):196-202.

17. Martin SS, Blaha MJ, Blankstein R, Agatston A, Rivera JJ, Virani SS, et al. Dyslipidemia, coronary artery calcium, and incident atherosclerotic cardiovascular disease: implications for statin therapy from the multi-ethnic study of atherosclerosis. Circulation. 2014;129(1):77-86.

18. Williams KA, Kim JT, Holohan KM. Frequency of unrecognized, unreported, or underreported coronary artery and cardiovascular calcification on noncardiac chest CT. J Cardiovasc Comput Tomogr. 2013;7(3):167-72.

19. Budoff MJ, Nasir K, Kinney GL, Hokanson JE, Barr RG, Steiner R, et al. Coronary artery and thoracic calcium on noncontrast thoracic CT scans: comparison of ungated and gated examinations in patients from the COPD Gene cohort. J Cardiovasc Comput Tomogr. 2011;5(2):113-8.

20. Pelandre GL, Sanches NMP, NacifMS, Marchiori E. Detection of coronary artery calcification with nontriggered computed tomography of the chest. Radiol Bras. 2018;51(1):8-12.

21. Hecht HS, Cronin P, Blaha MJ, Budoff MJ, Kazerooni EA, Narula J, et al. 2016 SCCT/STR guidelines for coronary artery calcium scoring of noncontrast noncardiac chestCT scans: a report of the Society of Cardiovascular Computed Tomography and Society of Thoracic Radiology. J Cardiovasc Comput Tomogr. 2017;11(1):74-84 